\title{
Parametric Studies about Vertical Position Control of the INTOR Elongated Plasma*
}

\author{
Koju Ueda, Satoshi Nishio ${ }^{\dagger}$, Masayoshi Sugihara ${ }^{\dagger}$, \\ and Noboru Fujisawa ${ }^{\dagger}$ \\ Mitsubishi Electric Corp., Tokyo 100.
}

(Received December 15, 1987)

\begin{abstract}
Considering only the most dominant of many eddy current modes induced in a fusion reactor, the authors estimated the shell effect required for suppression of the axisymmetric vertical positional instability of an elongated plasma. Here more accurate analysis involving higher eddy current modes is used. Also using this analysis, dependence of the shell effect and the plasma control characteristics upon the reactor structure is investigated. The parameters under consideration are shell shapes, number of the shells around the torus, and thickness of the shell structures for the shell effect. The delay time on a vertical position detection for the plasma, and the dead time of a power supply for the control coils are chosen as parameters for the feedback control system of the plasma position, too. Moreover, field penetration on the vertical position control into the plasma center is studied in order to show the appropriateness for the results in this paper.
\end{abstract}

Keywords :

active control, axisymmetric vertical positional instability, International Tokamak Reactor, passive control, shell effect, PID controller,

\section{Introduction}

In a recent paper(1), a method for suppression and control of the axisymmetric vertical positional instability of an elongated plasma was reported on the basis of conceptual design studies with the International Tokamak Reactor (INTOR).

The structure of INTOR there employed was composed of three kinds of inductive components, that is, 24 rectangular shells, 24 sectors of shields and a pair of control coils. The shell structure has been confirmed to be useful there. However on the analyses of the stabilizing effect expected from these components was used only the most dominant of many eddy current modes for each of the shells and the shields, and then the shell effect should be estimated more accurately.

* This work was done during the period ('80 '83) when K. Ueda joined the INTOR design team JAERI.

$\dagger$ Naka Fusion Research Establishment, Japan Atomic Energy Research Institute, Naka, Ibaraki 319-11. 
In this paper the following items will be presented.

(1) Vertical pcsition control involving higher eddy current modes is described on the basis of the same method of eddy current analysis as Reference (2), in which a thin conductor approximation and the finite element method are used.

(2) Parametric calculations are carried out for the reactor structure presented in the previous paper, naturally on taking higher eddy current modes into consideration. Shell shapes, number of the shells around the torus, and thickness of the shell structures are chosen as parameters for the shell effect. The delay time on a vertical position detection of the plasma, and the dead time of a power supply for the control coils are chosen as parameters for the feedback control system of the plasma position, too.

(3) Field penetration on the vertical position control into the plasma center is studied in order to obtain some evidences of the appropriateness for the results presented in this paper.

In the next section, basic considerations such as used assumptions, basic equations and analysis flows, will be given. In section 3 , a used reactor structure, the plasma parameters and obtained results are described. In section 4, these results are discussed on some points of view, that is, some results from the eddy current analysis using several groups of circular current loops, magnetic field penetration into the plasma center, and so forth.

\section{Basic Considerations}

\subsection{Assumptions}

In this paper, the plasma column is assumed to be equivalent to a circular current loop which has an infinitesimal cross-section and is placed at the same radial coordinate, $R_{P}(=$ the major radius) as the plasma center. Therefore the vertical equilibrium field, $B_{V}$ and the decay index, $n=\left(R / B_{V}\right)\left(\partial B_{R} / \partial Z\right)$, $R$ : radial coordinate, $\mathrm{Z}$ : axial coordinate, $\mathrm{B}_{\mathrm{R}}$ : radial equilibrium field used in the following analysis, are estimated at $R=R p$. Moreover the vertical displacement of the plasma is assumed to be so small that some change of the equilibrium field distribution can be neglected.

Also the following approximations are used here.

(1) The dipole approximation ${ }^{(1)}$ is used in estimation of the mutual inductances among the plasma column and the inductive components of INTOR.

(2) The inertial term in the equation of motion, by which the vertical motion of the plasma column can be represented, is negligibly small compared with the other terms in its equation.

(3) The eddy currents flowing in the inductive components of the reactor are estimated using the thin conductor approximation ${ }^{(2)}$.

\subsection{Basic equations}

The basic equations for the vertical position control of the plasma column consist of the equation of motion,

$$
Z_{p}=\left(n B_{v} / R_{p}\right) \cdot\left(\sum_{i=1}^{N} B_{i}+B_{d}\right)
$$

and the circuit equations, 


$$
\begin{aligned}
& \dot{I}_{i}+\sum_{\substack{j=1 \\
j \neq i}}^{N}\left(M_{i j} / L_{i}\right) \cdot \dot{I}_{j}+\left(R_{i} / L_{i}\right) \cdot I_{i} \\
= & \dot{Z}_{p} \cdot\left\{\left(\partial M_{p i} / \partial Z\right) L_{i}\right] \cdot I_{p}+\left(V_{i} / L_{i}\right) \quad(i=1, \cdots \cdots \cdots, N)
\end{aligned}
$$

where $Z_{P}$ and $\dot{Z}_{P}$ are the displacement of the plasma column in the vertical direction and its time derivative $\left({ }^{\bullet}=\mathrm{d} / \mathrm{dt}\right.$, t: time), respectively. $\dot{Z}_{\mathrm{P}}$ is given by the following equation.

$$
\dot{Z}_{p}=\left(n B_{v} / R_{p}\right) \cdot\left(\sum_{i=1}^{N} \dot{B}_{i}+\dot{B}_{d}\right)
$$

$I_{i}, L_{i}, R_{i}, M_{i j}$ and $M_{p i}$ with the $i-t h$ inductive component are its current, self-inductance, resistance, and mutual inductances for the $j$-th component and the plasma column, respectively. Here the number of the inductive components is $\mathrm{N}$ of which $\mathrm{N}-1$ is the number of the eddy current modes indicated by the suffixes, 1, 2, ---, $(\mathrm{N}-1)$, and the suffix, $\mathrm{N}$ is used for a pair of control coils. $\mathrm{Bi}_{\mathrm{i}}(\mathrm{i}=1,---, \mathrm{N})$ can be expressed by the following equations.

$$
B_{i}=\nu_{i} \cdot I_{i}(i=1, \cdots \cdots \cdots, N)
$$

where $\nu_{i}$ is the radial field per unit current produced at $\left(R_{p}, 0.0\right)$ by the $i-t h$ inductive component or the control coils.

$\mathrm{B}_{\mathrm{d}}$ is the disturbance field at $\left(\mathrm{R}_{\mathrm{P}}, 0.0\right)$ causing a vertical motion of the plasma column, and is given by the following.

$$
B_{d}=B_{0} \cdot\left[1-\exp \left(-t / \tau_{d}\right)\right\rfloor
$$

where $\mathrm{B}_{0}$ and $\tau_{\mathrm{d}}$ are the disturbance field at $\mathrm{t} \rightarrow \infty$ and the time constant, respectively. $V_{I}$ is zero except for the control coils $(i=N)$, and $V_{n}$ is the voltage between the coil's feeders and is given as follows:

$$
\begin{aligned}
V_{n}= & -G \cdot\left[Z_{p}\left(t-T_{\text {dead }}\right)+t_{D} \cdot \dot{Z}_{p}\left(t-T_{\text {dead }}\right)\right. \\
& +\left(1 / t_{I}\right) \cdot \int_{0}^{\left.t-T_{d e a d} Z_{p} d t\right]}
\end{aligned}
$$

where $G, T_{\text {dead }}, t_{r}$, and $t_{I}$ are the gain, the dead time of the power supply, the differential time and the integral time of the PID controller, respectively. It is set so as to be $Z_{p}=0$ in Eq. (6) when $t \leqq 0$.

Detection of the vertical position of the plasma column is necessarily accompanied with a delay time. Here the first order delay given by the following eqs. is assumed for the delay of its detection.

$$
\begin{aligned}
\left(Z_{p}^{*}\right)_{m} & =\left(Z_{p}^{*}\right)_{m-1} \cdot \exp \left(-\Delta t / T_{\text {delay }}\right) \\
& +\left(Z_{p}\right)_{m} \cdot\left[1-\exp \left(-\Delta t / T_{\text {delay }}\right)\right],
\end{aligned}
$$




$$
\begin{aligned}
\left(\dot{Z}_{p}^{*}\right)_{m} & =\left(\dot{Z}_{p}^{*}\right)_{m-1} \cdot \exp \left(-\Delta t / T_{\text {delay }}\right) \\
& +\left(\dot{Z}_{p}\right)_{m} \cdot\left[1-\exp \left(-\Delta t / T_{\text {delay }}\right)\right],
\end{aligned}
$$

where $T_{\text {delay }}$ is the delay time whose value is defined here so as to be the same as for both of $Z_{\mathrm{P}}$ and $\dot{Z}_{\mathrm{P}} .\left(Z_{\mathrm{P}}\right)_{\mathrm{m}}$ and $\left(\dot{Z}_{\mathrm{P}}\right)_{\mathrm{m}}$ are the input for the first order delay circuit and $\left(Z_{P}{ }^{*}\right)_{m}$ and $\left(\dot{Z}_{P}{ }^{*}\right)_{m}$ are the output from the circuit.

The suffixes, $m$ and $(m-1)$ mean them at $t$ (time under consideration) and $\mathrm{t}-\Delta \mathrm{t}$ (before $\mathrm{t}$ by $\Delta \mathrm{t}$ ), respectively, when a series of eqs., (1) -(3), are numerically solved using an iteration technique.

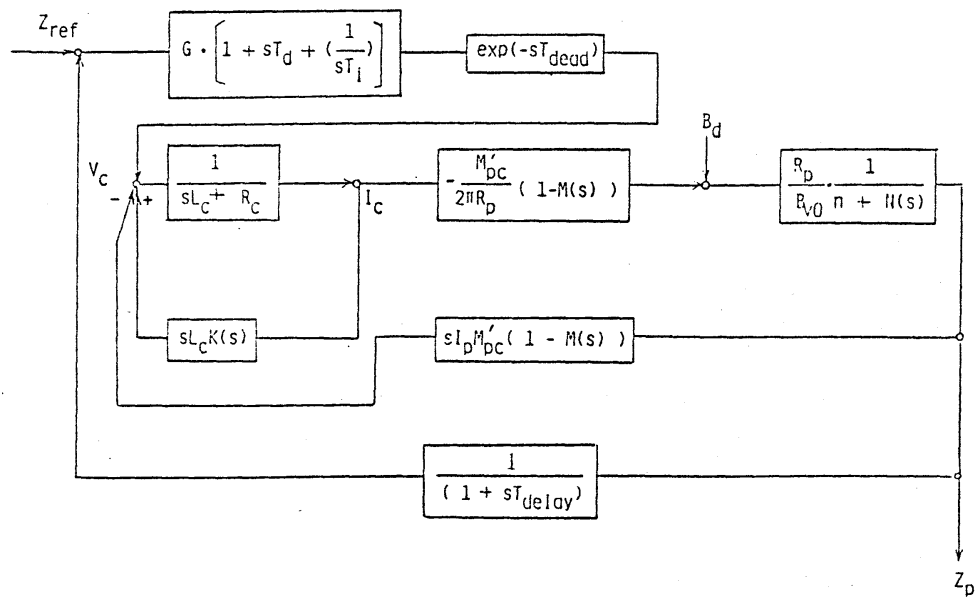

Fig. 2. 1 Block diagram of the vertieal position control system.

\subsection{Used control system}

The block diagram of the vertical position control system which is used here, is shown in Fig. 2.1, and is almost the same construction as one proposed in Reference (2). The symbols in the figure are defined as follows:

$$
\begin{aligned}
K(s) & =\sum_{i} K_{i} \cdot s \cdot \tau_{i} /\left(1+s \cdot \tau_{i}\right), \\
M(s) & =\sum_{i} \mu_{i} \cdot s \cdot \tau_{i} /\left(1+s \cdot \tau_{i}\right), \\
N(s) & =\sum_{i} n_{i} \cdot s \cdot \tau_{i} /\left(1+s \cdot \tau_{i}\right), \\
K_{i} & =M_{N i}{ }^{2} / L_{N} \cdot \tau_{i} \\
\mu_{i} & =M_{N i}^{2} \cdot M_{p i}^{\prime} / M_{p N} \cdot \tau_{i}
\end{aligned}
$$




$$
\begin{aligned}
& n_{i}=-M_{p i}^{\prime} \cdot I_{p} / 2 \pi B_{v} \cdot \tau_{i} \\
& \nu_{i}=-M_{p i}^{\prime} / 2 \pi R_{p} \\
& (i=1,2, \cdots, N-1, N), \\
& N_{N}(s)=n_{N} \cdot s \cdot \tau_{N} \cdot\{1-M(s)\}^{2} /\left[1+s \cdot \tau_{N} \cdot\{1-K(s)\},\right. \\
& n_{N}=-M_{p N}^{2} \cdot I_{p} / 2 \pi B_{v} L_{N}, \\
& \tau_{N}=L_{N} / R_{N}, \\
& \nu_{N}=-M_{p N}^{\prime} / 2 \pi R_{p},
\end{aligned}
$$

where the suffixes are the same as in section 2.2, and 'denotes the derivative with respect to $Z$, that is, $\partial / \partial Z$.

The transfer function for the open loop is given by the following:

$$
\begin{aligned}
A(s) & =G \cdot\left\{\left(1+s t_{D}\right)+\left(1 / s t_{I}\right)\right\} \\
& \cdot\left\{F(s) \cdot e^{-s} T_{\text {dead }} /\left(1+s T_{\text {delay }}\right)\right\},
\end{aligned}
$$

where $F(s)$ is defined as follows:

$$
\begin{aligned}
F(s)= & \left(R_{p} \tau_{N} \nu_{N} / B_{v} L_{N}\right) \cdot\{1-M(s)\} \\
& /\left\{n+N(s)+N_{N}(s)\right\} \cdot\left[1+s \cdot \tau_{N} \cdot\{1-K(s)\}\right],
\end{aligned}
$$

The shell effect can be expected if the following condition is satisfied.

$$
n+N(\infty)+N_{N}(\infty)>0
$$

Moreover, when the shell effect can be expected, the growth rate of the vertical positional instability, $\gamma$ is given by the following equation:

$$
n+N\left(r_{g}\right)+N_{N}\left(r_{g}\right)=0
$$




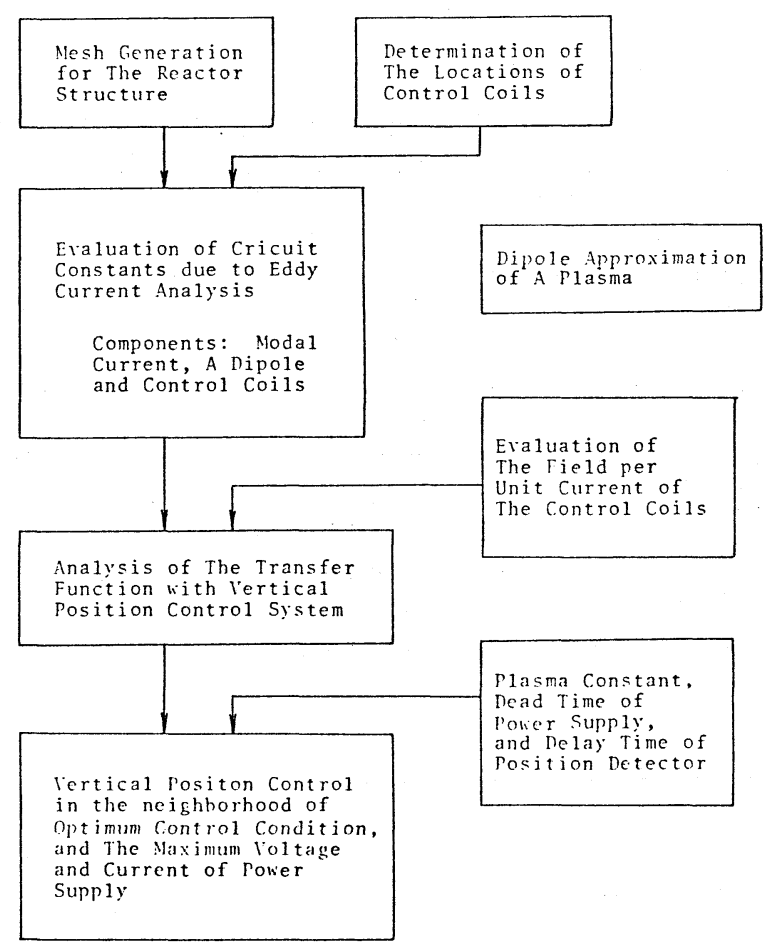

Fig. 2.2 A typical calculation process for evaluation of the capacity of power supply.

\subsection{Caluculation process}

In Fig. 2.2 is shown a typical calculation process, that is, an application to determination of the capacity of the power supply used for the vertical position control coils. On the process are used three kinds of codes: (1) an eddy current code, (2) a transfer function analysis code, and (3) a vertical position control analysis code.

Table 3. 1 Main Parameters of the INTOR plasma

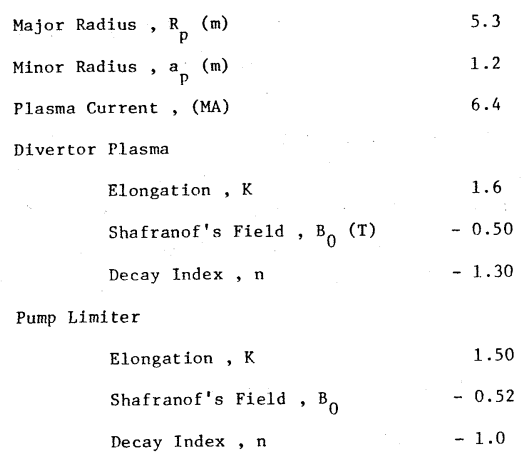

Prior to the eddy current calculation, the modelled reactor structure is divided into finite triangle elements, and its mesh generation is completed. Then, the eddy current calculation is carried out, and the circuit constant required for both analyses of the transfer function and the vertical poisition control are determined. The transfer function is analyzed through the Nyquist diagram and the amplitude-phase diagram. Consequently some conditions required for the stable control of the vertical positional instability will be clarified. 


\section{Results}

\subsection{Modelling of reactor structure}

Main parameters of the INTOR plasma, $R_{P}, r_{P}, I_{P}, B_{V}$ and $n$ which are defined in section 2.2 and concretely shown on Table 3.1 are selected to be equal to $5.3 \mathrm{~m}, 1.2 \mathrm{~m}, 6.4 \mathrm{MA},-0.5 \mathrm{~T}$ and -1.3 , respectively. As shown in the previous paper(1), the plasma has asymmetey between its lower part and upper part, and its magnetic flux configuration is a single null divertor type. Therefore, the reactor structure of INTOR will be constituted to have asymmetry between them, too. However, in this paper is assumed the structure which is symmetrical with regard to the median plane (axial coordinate, $Z=0$ ) as shown in Fig. 3.1, in order to facilitate both the applications of the finite element method and the eddy current analysis to one. A thin conductor structure modelled for Fig. 3.1 is shown in Fig. 3.2 (a), and also the structures of the shell-I and -(I+ II) are given in Fig. 3.2 (b) and (c), respectively. Each figure shows a half of one sector on the upper side of the modeled reactor, and the toroidal angle of one sector is equal to $360^{\circ} /$ the number of sectors into which the torus are divided in the toroidal direction.

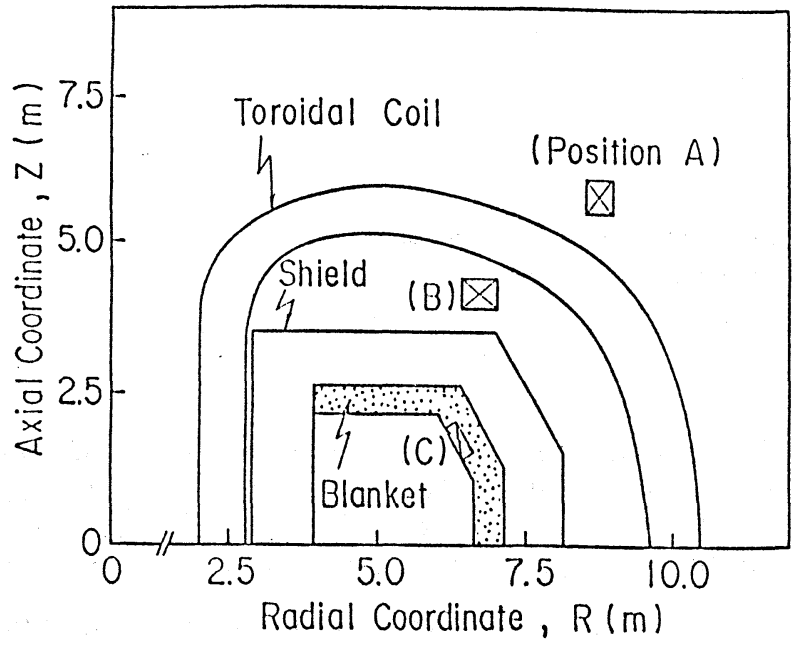

Fig. 3. 1 Vertical cross-sectional view of used reactor structure.

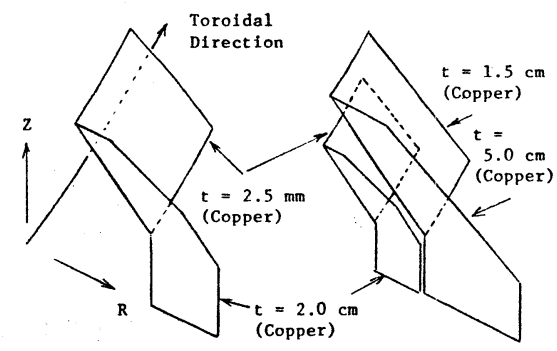

(b) She11-I

(c) Shel1- $(1+$ II)

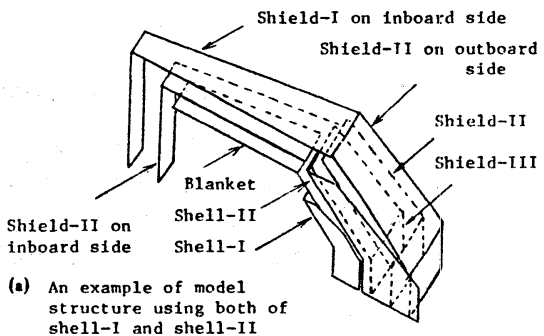

Fig. 3.2 A modelled structure of the reactor: (a) An example of the structure using both of shell-I and-II, (b) shell- I and (c) shell-( I + II ).

As shown in Fig. 3.2 (a), the reactor is composed of the shell-I and -II, the shield -I, -II and -II on the outboard side, the shield-I and -II on the inboard side and the blankets. There are not included the vacuum chamber and the others. In this paper, it is assumed that the shells are made of copper, and the shields and the blankets are done of stainless steel. Their values in thickness are assumed as follows:

\section{Shell-I (toroidal plate)}

Shell-I (poloidal plate)
$2.5-10.0 \mathrm{~mm}$

$20.0 \mathrm{~mm}$ 


$\begin{array}{ll}\text { Shell-II (toroidal plate) } & 15.0 \mathrm{~mm} \\ \text { Shell-II (poloidal plate) } & 50.0 \mathrm{~mm} \\ \text { Shield-I (inboard side) } & 400 \mathrm{~mm} \\ \text { Shield-II (inboard side) } & 400 \mathrm{~mm} \\ \text { Shield-I (outboard side) } & 300 \mathrm{~mm} \\ \text { Shield-II (outboard side) } & 400 \mathrm{~mm} \\ \text { Shield-II (outboard side) } & 300 \mathrm{~mm} \\ \text { Blanket } & 50 \mathrm{~mm}\end{array}$

As found from the above values, only the thickness of two toroidal plates of the shell-I is shown over a range of $2.5-10.0 \mathrm{~mm}$. The effect of its thickness on the instability growth time (Shell Effect) are parametrically studied over the range.

Also the number of the toroidally arranged sectors is selected as the other parameter for parametrical studies of the shell effect.

The structure of Fig. 3.2 (a) will be found to content itself with the following requirements for the INTOR design in order to facilitate its remote assembly and maintenance.

(1) Poloidal field coils should be located outside the toroidal field coils.

(2) Portwindows should have several meters in vertical width on both the upper and and lower parts of the mechanical midplane outside the toroidal coils.

(3) Shell structures and shields should be toroidally divided in to some dozens of sectors.

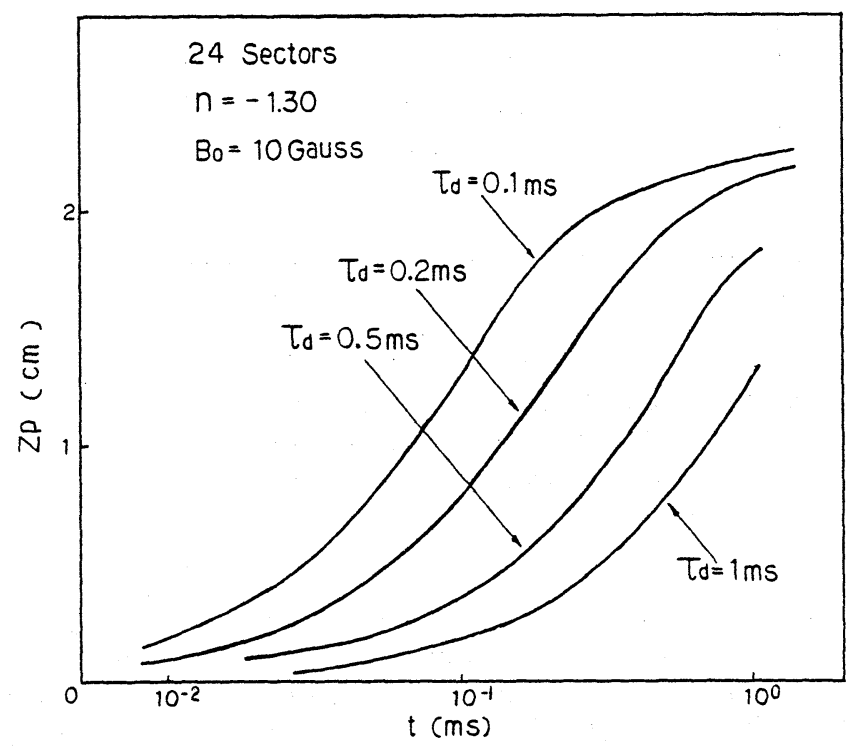

Fig. 3. 3 Time evolutions of the vertical displacement, $Z_{p}$, when the disturbance of eq. (5) is imposed on around the plasma column. 


\subsection{Shell effect}

Figure 3.3 shows time evolutions of the vertical displacement of the plasma column, $Z_{P}$, when the external horizontal disturbance field of Eq. (5) is imposed on around the column. In the figure, Bo is selected 10 Gauss and $\tau_{d}$ is stepwise varied from $0.1 \mathrm{~ms}$ to $1.0 \mathrm{~ms}$. The number of the toroidally arranged sectors, and the decay index are fixed at 24 and -1.3 respectively, and these values were adopted as a reference of INTOR. In the case of $\tau_{d}=0.1$ msec, the value of $Z_{P}$ grows abruptly with time, and its variation is found to convert a slow growth rate after $0.5 \mathrm{~ms}$. Such a conversion is observed in the others of $\tau_{\mathrm{d}}$, and these growth rates after the conversions are found to be nearly equal to a constant value, which is about $(1 / 35.7)(\mathrm{ms})^{-1}$ in the case of Fig. 3.3 and independent upon $\tau_{d}$. The constant value is a peculiar value to the shell structure, and is of use as a measure of the shell effect with it.

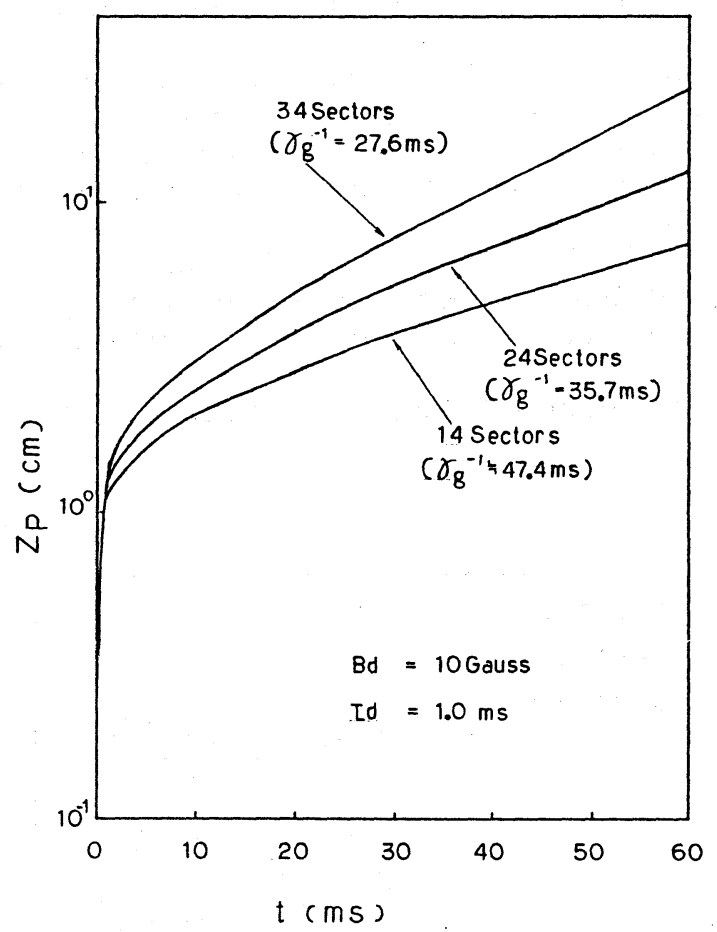

Fig. 3. 4 Time evolutions of $Z_{p}$ in $\tau_{d}=1.0 \mathrm{~ms}$ and $B_{0}=10$ Gause, where the number of the toroidally arranged sectors is selected as a parameter.

Figure 3.4 shows time evolutions of $Z_{P}$ in $\tau_{d}=1.0 \mathrm{~ms}$ and $B_{0}=10$ Gauss, where the number of the sectors toroidally arranged on the full torus is selected as a parameter. The growth times for three kinds of numbers, that is, 14, 24 and 34 , are searched for and found to be approximately $47.4,35.7$ and 27.6 ms, respectively.

Figure 3.5 shows variations of the growth time vs. the thickness of two plates placed along the toroidal direction on the upper and lower parts of shell- 

the INTOR Elongated Plasma

I. In this figure, the number of the sectors is fixed at 24 . The notation "shell-I" means the shell effect given only by the shell structure of Fig. 3.2 (b), and also "shell-(I+ II )" does one by the shell structure of Fig. 3.2 (c), without the other reactor structures. The other cases show the shell effect with the reactor structure given in Fig. 3.2 (a).

The growth time is found to increase with increasing the thickness of the toroidal plates, and such an inclination is in common with four cases. Also the other inductive structures, that is, the shields and the blankets, are found to make the shell effect more effective than one without them.

On the other hand the shell effect corresponding to $2.5 \mathrm{~mm}$ in the thickness of these toroidal plates was reported on the previous paper, and then was about $21 \mathrm{~ms}$ in growth time for a simplified reactor structure with the shell-I, and moreover was about $34.8 \mathrm{~ms}$ for one with the shell-(I+ II).

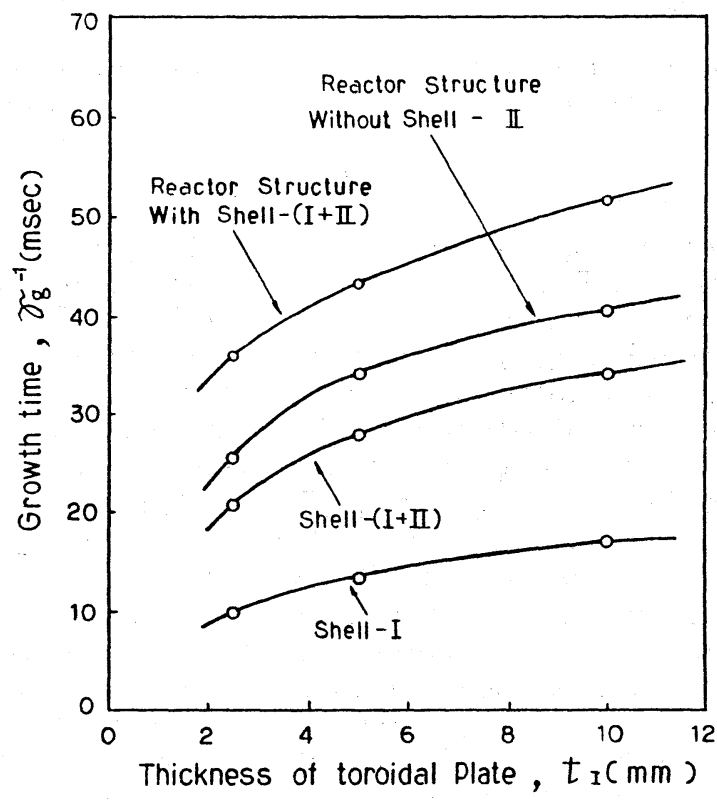

Fig. 3. 5 Variations of the growth time vs. the thickness of two plates placed along the toroidal direction on the upper and lower parts of shell-I.

It is found from Fig. 3.5 that these values of the growth time are in good agreements with the results of this report for the reactor structure without and with the shell- II. Therefore, the previous analysis using only the most dominant of many eddy current modes for each of the shells and the shield, is found to give sufficient results with little error for estimation of the shell effect. 


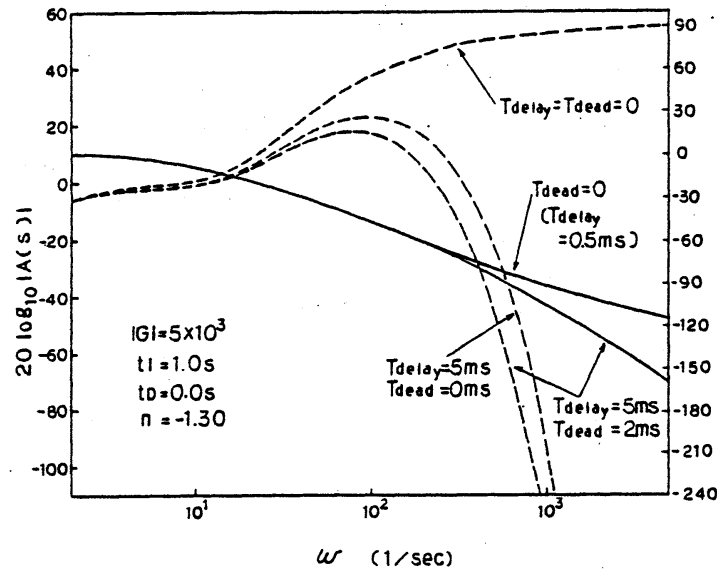

Fig. 3.6 (a) An amplitude and phase diagram for the PI controller.

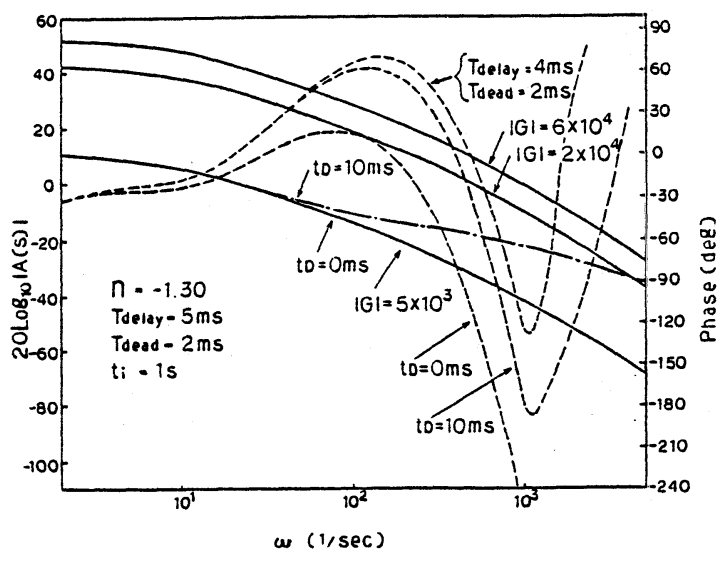

Fig. 3.6 (b) An amplitude and phase diagram for the PID controller.

\subsection{Control characteristics}

At first, the open loop transfer function, A(s) given by Eq. (20) is studied, and the results shown in Figs., 3.6 (a) and (b) are obtained. Fig. 3.6 (a) is an amplitude and phase diagram for the PI controller. The delay time of the plasma position detection, $T$ delay, and the dead time of the power supply of control coils, $T$ dead are selected there as parameters. The amplitude and the phase in the figure are indicated by solid lines and dotted lines, respectively. The PI controller is found to give a stable system if $T_{\text {delay }}=T_{\text {dead }}=0$, and on the other hand, to become unstable if $T_{\text {delay }}=5 \mathrm{~ms}$ and $\mathrm{T}_{\text {dead }}=2 \mathrm{~ms}$ whose conditions will be actually faced on used devices.

Fig. 3.6 is one in case of the PID controller. The dotted lines indicate the phase variations, and the solid lines do the amplitude variations in which the gain, $G$ is choiced as a parameter and the differential time, $t_{D}$ is fixed at $0 \mathrm{~ms}$. A dot and dash line shows the amplitude variation in which only $t_{D}$ is changed into $10 \mathrm{~ms}$ under the same condition as one with the solid lines.

It is found from Fig. 3.6 (b) that, if $\mathrm{T}_{\text {delay }}=5 \mathrm{~ms}$ and $\mathrm{T}_{\text {dead }}=2 \mathrm{~ms}$, the phase angle at $10^{3}(1 / \mathrm{sec})$ of $\omega$ becomes a bit less than $-180^{\circ}$, and moreover, if $\mathrm{T}_{\text {delay }}=4 \mathrm{~ms}$ and $\mathrm{T}_{\text {dead }}=2 \mathrm{~ms}$, the stable one whose phase margin is more than 30 degrees is obtained.

A typical characteristics of the position control system in case of $\mathrm{T}_{\text {dead }}=$ $\mathrm{T}_{\text {delay }}=2 \mathrm{~ms}$ is shown in Fig. 3.7, where time evolutions of the axial position of the plasma, $Z_{P}$, the voltage, $V_{c}$ and the current, $I_{c}$ of the power supply, and the magnetic field due to induced eddy current, $B_{\text {eddy }}$ are given. $Z_{P}$ is found to attain the maximum at about $3 \mathrm{~ms}$, and then, to stabilize after a slight fluctuation. $V_{c}$ begins to increase rapidly after $3 \mathrm{~ms}$, attains the maximum value, $2.0 \mathrm{kv}$ at $4.7 \mathrm{~ms}$, and then is attenuated. On the other hand, $\mathrm{I}_{\mathrm{c}}$ reaches the first peak at $6 \mathrm{~ms}$, gets the second peak (the maximum), $0.14 \mathrm{MA}$ at 20 $\mathrm{ms}$, and then is stabilized. $300 \mathrm{MVA}$ in the product of the maximum voltage and the maximum current is approximately required for the power supply in this case. This capacity of the power supply is found to be more than one on the previous paper from one order to two. 


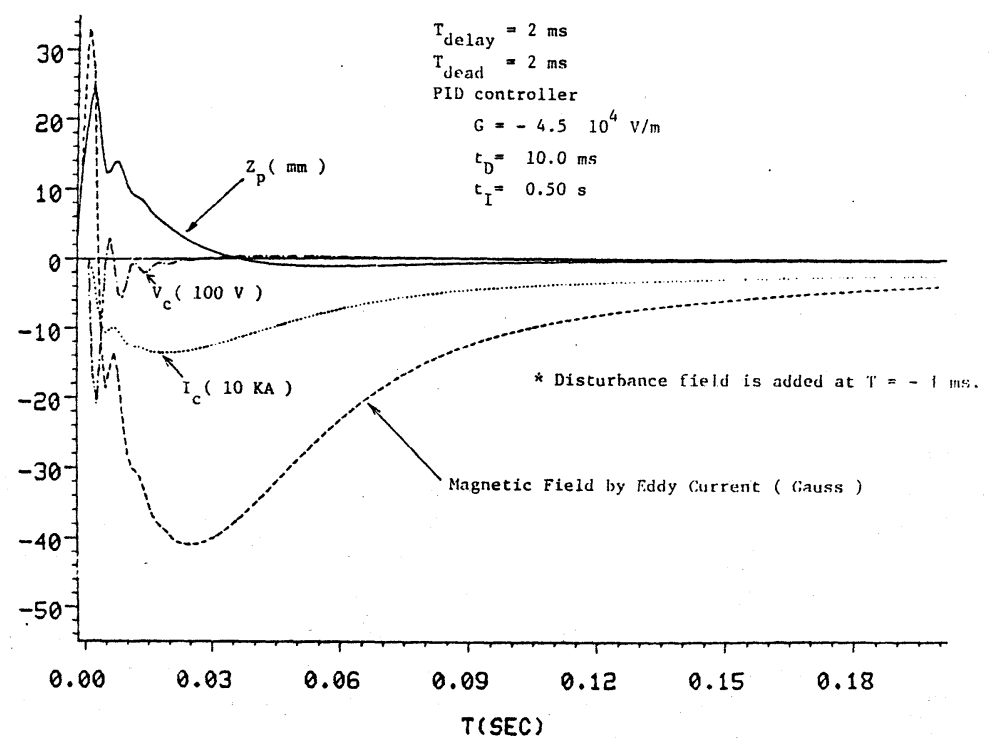

Fig. 3.7 A typical characteristics of the position control system in case of $T_{\text {dead }}=T_{\text {delay }}=2 \mathrm{~ms}$.

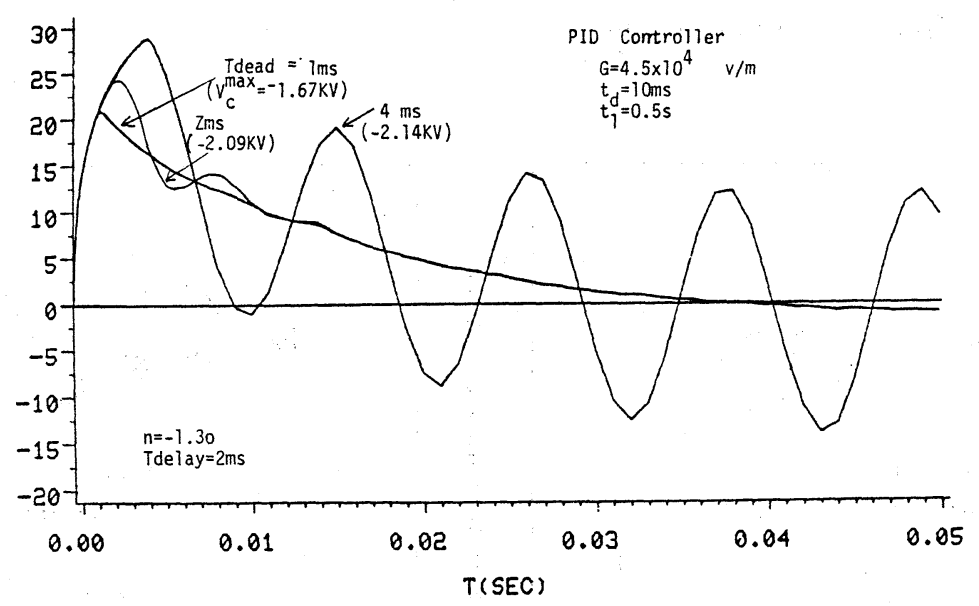

Fig. 3. 8 Time evolutions of $Z_{p}$ for three kinds of $T_{\text {dead }}$ values, that is, $1.0,2.0$ and $4 \mathrm{~ms}$, and $T_{\text {delay }}$ fixed at $2 \mathrm{~ms}$.

In Fig. 3.8 are shown time evolutions of $\mathrm{Z}_{\mathrm{P}}$ for three kinds of $\mathrm{T}_{\text {dead }}$ values, that is, $1.0,2.0$ and $4 \mathrm{~ms}$. $T$ delay is fixed at $2 \mathrm{~ms}$. With decreasing $\mathrm{T}_{\text {dead }}$ the maximum value of $\mathrm{Z}_{\mathrm{P}}$ decreases, and more stable control is obtained when $T_{\text {dead }}=1.0 \mathrm{~ms}$. Then the required product is approximately equal to 230 MVA. 


\section{Discussions and Conclusions} Fig. 3.5.

Dependence of the shell effect upon the following four cases is shown in

(1) With shell-I only

(2) With shell- $(\mathrm{I}+\mathrm{II})$

(3) Reactor structure with shell-I and without shell-II

(4) Reactor structure with shell-(I+I)

The structure employed in the previous paper(1) was composed of three kinds of inductive components, that is, 24 rectangular shells, 24 sectors of shields and a pair of control coils, too. Difference between this paper's and the previous results is found to be negligibly small for both of (3) and (4). Judging from these results, the analysis using only the most dominant of many eddy current modes with each of the inductive components of the reactor is found to give almost the same growth time as that taking higher eddy current modes into consideration. Therefore, whether or no noticed inductive component is effective for the shell effect is known from that of the most dominant eddy current mode with it.

The above-mentioned results are obtained from the eddy current analyses based on both methods of the thin plate approximation and the finite element. Here these will be checked by the more simple and broadly known method ${ }^{(3)}$, that is, a method based on the partition of a structure into several groups of circular current loops whose central axes are arranged coaxially with its symmetical axis $(\mathrm{R}=0)$. This method is called "method 2" for the "method 1 " which is based on the finite element method.

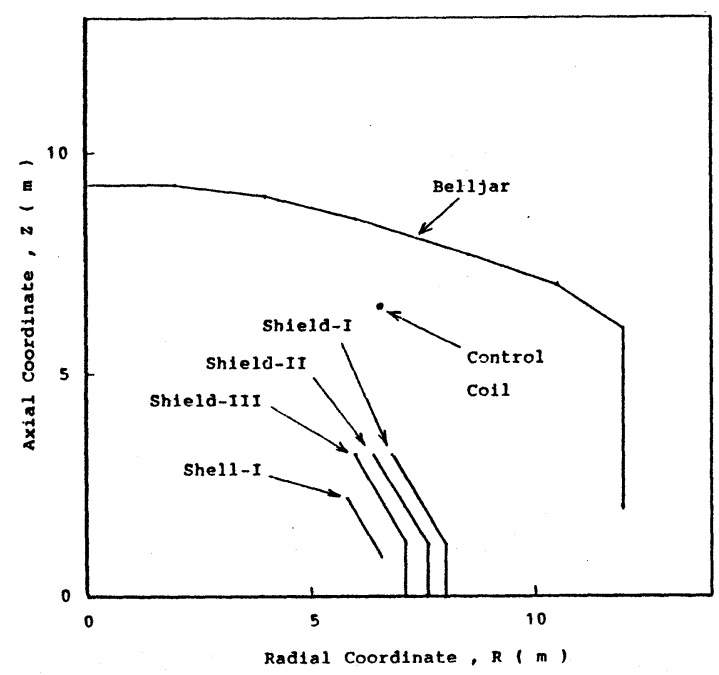

Fig. 4. 1 Vertical cross-sectional view of inductive components around plasma used in section 4.

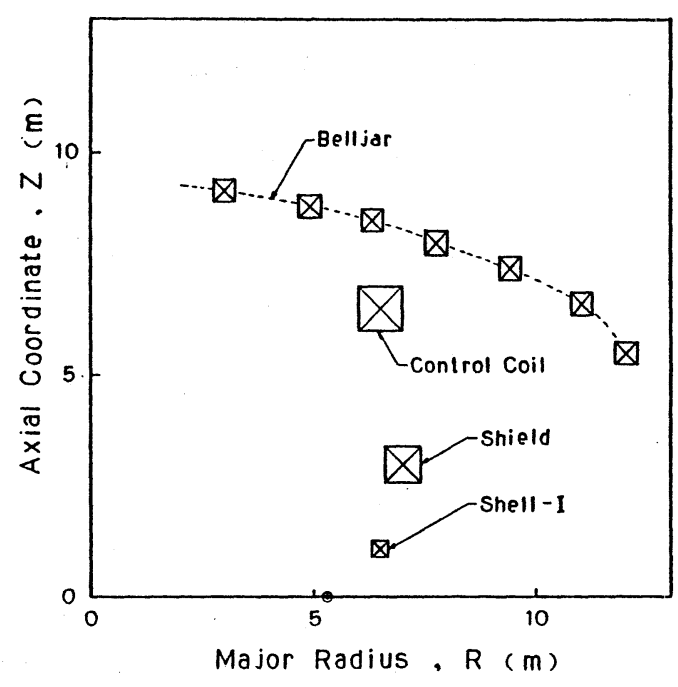

Fig. 4.2 Several groups of circular coils used for the "method 2 ". 
The reactor structure here used, is shown in Fig. 4.1, and is composed of the shell-I $\left(t_{r}=10 \mathrm{~mm}\right.$ in copper thickness), radiation shields (three layers of stainless steel plates, $3 \times 300 \mathrm{~mm}$ in total thickness), and belljars $(50 \mathrm{~mm}$ in stainless steel thickness). This structure doesn't have any cut in the toroidal direction other than the shell-I, and therefore can be approximated by several groups of circular current loops except for the shell-I. Of course, the shell-I will be approximately replaced by a pair of circular current loops, too. Several groups of circular coils used for the "method 2" are shown in Fig. 4.2. Their circuit constants are given on Table 4.1, where $\mathrm{Mij}$ is the mutual inductance between the $i$ coil and the $j$ one in $\mu \mathrm{H}$, and $r_{j}$ is the resistance of the $j$ coil in mohm. No.10 on Table 4.1 means a pair of control coils, the numbers from No.1 to No.7 are used for the belljar, No.8 is the shell-I, and No.9 is the shields. Coil cross-sections from No.1 to No.9 are set to be equal to $0.5^{\mathrm{m}} \mathrm{x}$ $0.10^{\mathrm{m}}$ for all coils. On the other hand, Fig. 4.3 shows several groups of finite elements used for the "method 1". There is presented one twenty fourth of the reactor structure shown in Fig. 4.1.

Table 4. 1 Circuit Constants of Inductive components.

\begin{tabular}{|c|c|c|c|c|c|c|c|c|c|c|c|c|c|}
\hline \multirow{2}{*}{$j i$} & \multicolumn{10}{|c|}{$M_{11}$} & \multirow{2}{*}{$r_{j}$} & \multirow{2}{*}{$M_{p j}^{\prime}$} & \multirow[b]{2}{*}{$\nu_{j}$} \\
\hline & 1 & 2 & 3 & 4 & 5 & 6 & 7 & 8 & 9 & 10 & & & \\
\hline 1. & 22.38 & 8.39 & 5.87 & 4.37 & 3.27 & 2.46 & 1.89 & 0.46 & 1.45 & 4.11 & 0.26 & 0.19 & -0.06 \\
\hline 2 & 8.39 & 42.30 & 20.14 & 13.43 & 9.58 & 7.04 & 5.36 & 1.18 & 3.78 & 11.85 & 0.43 & 0.45 & -0.13 \\
\hline 3 & 5.87 & 20.14 & 57.29 & 27.29 & 17.75 & 12.62 & 9.48 & 1.78 & 5.87 & 19.43 & 0.56 & 0.64 & -0.19 \\
\hline 4 & 4.37 & 13.43 & 27.29 & 74.30 & 33.31 & 21.80 & 15.95 & 2.40 & 8.08 & 23.29 & 0.68 & 0.81 & -0.25 \\
\hline 5 & 3.27 & 9.58 & 17.75 & 33.31 & 92.82 & 41.10 & 27.82 & 2.80 & 9.43 & 18.94 & 0.83 & 0.89 & -0.27 \\
\hline 6 & 2.46 & 7.04 & 12.62 & 21.80 & 41.10 & 109.82 & 51.20 & 2.811 & 9.27 & 14.14 & 0.97 & 0.85 & -0.25 \\
\hline 7 & 1.89 & 5.36 & 9.48 & 15.95 & 27.82 & 51.2017 & 117.90 & 2.70 & 8.64 & 11.03 & 1.06 & 0.78 & -0.24 \\
\hline 8 & 0.46 & 1.18 & 1.78 & 2.40 & 2.80 & 2.81 & 2.70 & 40.84 & 10.41 & 3.13 & 0.54 & 5.76 & -1.77 \\
\hline 9 & 1.45 & 3.78 & 5.87 & 8.08 & 9.43 & 9.27 & 8.64 & 10.41 & 58.62 & 10.52 & 0.62 & 3.05 & -0.92 \\
\hline 10 & 4.11 & $11.85 \mid$ & 19.43 & 23.29 & 18.94 & 14.14 & 11.03 & 3.13 & 10.52 & 59.34 & - & 1.13 & -0.34 \\
\hline
\end{tabular}

Figure 4.4 shows both time evolutions of the surface current integral over all inductive components obtained by the "method 1", and the sum of coil currents from No.1 to No.9 by the "method 2" in the case where the control coil current flows in a form, $I_{0}\left[1-\exp \left(-t / \tau_{c}\right)\right]\left(t:\right.$ time, $\tau_{c}=$ time constant, $I_{0}$ : control coil current on $t=\infty)$.

Difference between two current sums is found to be little in the phase of rising current, as inductances with the inductive components can be faithfully evaluated for both of the "methods, 1 and 2 ". On the other hand, the difference is large in the phase of decreasing current as the resistance of each coil in the "method 2" couldn't be determined so as to give the same effect as distributed resistances in the "method 1". Notwithstanding such a defect, each current sum arrives a peak at $30 \mathrm{~ms}$ in the elapsed time, though difference between both peak currents remains as much as $10 \%$. Judging from these results, the "method 2" in the rising current phase is found to give almost the same inclination as the "method 1".

Figure 4.5 shows time evolutions of the horizontal magnetic fields at the plasma center $(R=5.3 \mathrm{~m}, Z=0 \mathrm{~m})$ for two "methods, 1 and $2 "$. As a whole, the result from the "method 2" are in good agreements with one in the "method 


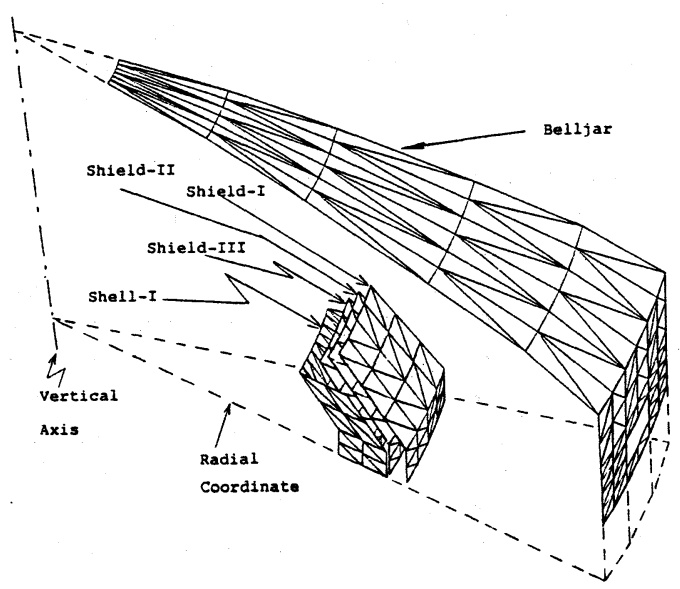

Fig. 4.3 Several groups of finite elements used for the "method 1 ".

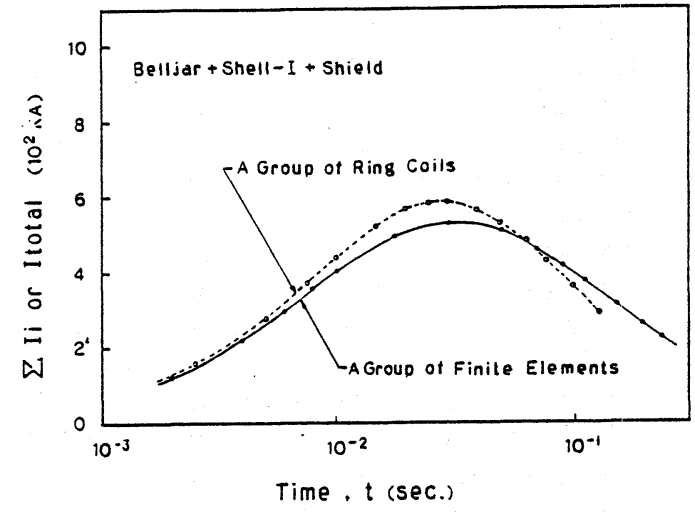

Fig. 4.4 Both time evolutions of the surface current integral over all inductive components by the "method 1 ", and the sum of coil currents from No. 1 to No. 9 by the "method 2 " on the current flow given by $I_{0}(1$ $\left.\exp \left(-t / \tau_{0}\right)\right)$.

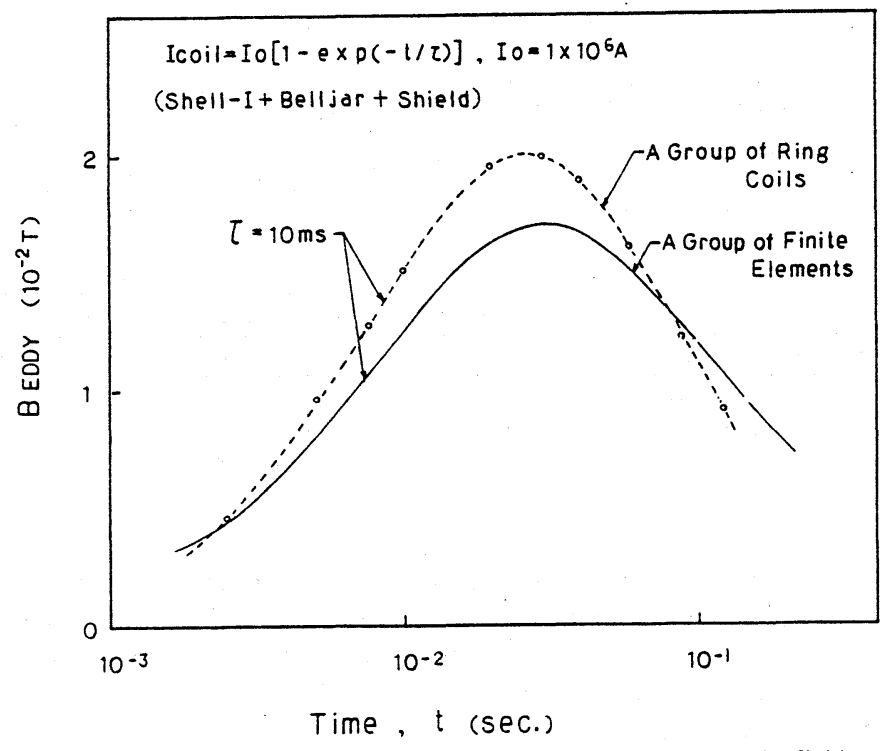

Fig. 4.5 Time evolutions of the horizontal magnetic fields at the plasma center $(R=5.3 \mathrm{~m}, Z=0 \mathrm{~m})$ for two "methods, 1 and 2 ".

$1 "$ in Fig. 4.5 as well as in Fig. 4.4.

Such an agreement is thought to be an evidence of the appropriateness for used "methods, 1 and 2" though it is obtained only in the rising current phase. As shown in Fig. 3.7 the vertical position of the plasma is found to pass through zero in the vertical coordinate at about $35 \mathrm{~ms}$, and to go toward the target position (zero) gradually after about $50 \mathrm{~ms}$ in the elapsed time where its position has the minimum value. On the other hand, the magnetic field due to the eddy current arrives a negative peak at about $25 \mathrm{~ms}$, and are slowly damped 
after that time. To discuss the appropriateness for these results, penetration of the magnetic flux due to a pair of control coils to the plasma region is studied using the above-mentioned "method 1 ".

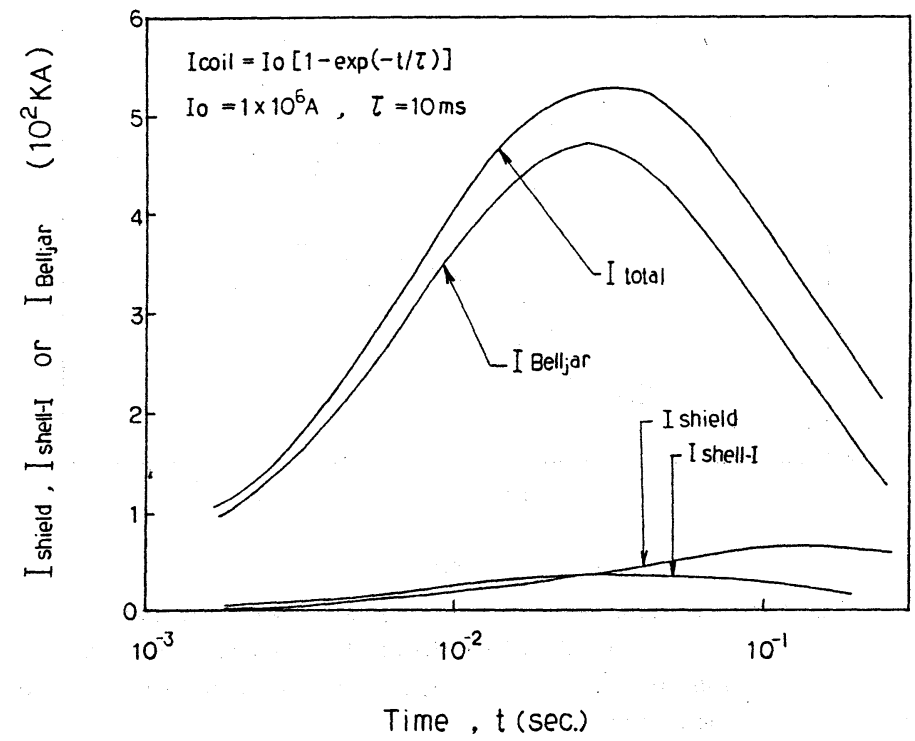

Fig. 4. 6 Time evolutions of the eddy currents flowing on the reactor components, the belljar, the shields and shell- I .

Figure 4.6 shows time evolutions of the eddy currents flowing on the reactor components, that is, the belljar, the shields and the shell-I. Each current is found to have a peak at the elapsed time, which is about $30 \mathrm{~ms}$ for the belljar, about $150 \mathrm{~ms}$ for the shields, or about $25 \mathrm{~ms}$ for the shell-l. The current integral shows a current pattern which is similar to the belljar's one.

Figure 4.7 shows time variations of both magnetic fields due to the control coils $\left(\mathrm{B}_{\text {coil }}\right)$ and the total eddy current $\left(\mathrm{B}_{\text {eddy }}\right)$, and $\delta B\left(=|| \mathrm{B}\right.$ coil $\left.|-| \mathrm{B}_{\text {eddy }}||\right)$ at the major radius of the plasma. $\delta B$ is the absolute value of the remainder between $\left|B_{\text {coil }}\right|$ and $\left|B_{\text {eddy }}\right|$. In the figure, the time constant of the coil current, $\tau_{c}$ is selected to be equal to $10 \mathrm{~m} \mathrm{sec}$. The magnetic field, $B$ coil attains $0.5 \mathrm{Bo}(\mathrm{B}$ coil at $t=\infty)$ after $7 \mathrm{~ms}$, and $0.95 \mathrm{Bo}$ after $30 \mathrm{~ms}$. On the other hand, $B$ eddy increases monotonously by the peak value, which occurs at about $30 \mathrm{~ms}$. The peak value is equal to about $0.53 \mathrm{~B}$ coil $(0.5 \mathrm{Bo})$, and therefore the remainder of $\mathrm{Bo}, 0.47 \mathrm{~B}$ coil $(0.45 \mathrm{Bo})$ is found to penetrate to the region of the plasma. $\delta \mathrm{B}$ is equal to $\mid \mathrm{B}$ eddy $\mid$ at $40 \mathrm{~ms}$ and attains $60 \%$ of $\mid \mathrm{B}$ coil $\mid$ at $100 \mathrm{~ms}$. Moreover, as known from Fig. 4.7, $\delta \mathrm{B}$ at $2 \mathrm{~ms}, 5 \mathrm{~ms}$ and $10 \mathrm{~ms}$ is found to attain $39 \%, 40 \%$ and $42 \%$ of |B coil|, respectively, and about $40 \%$ of $\mid \mathrm{B}$ coillis known to penetrate to the region of the plasma in a few $\mathrm{ms}$ of the elapsed time. Judging from these results, the control coils are needed to supply 2.5 times the magnetic flux which is required for stabilizing the vertical position of the plasma in a time range, $2-30 \mathrm{~ms}$.

When the plasma displacement, $Z_{P}$ is given, the magnetic field with which the plasma counterbalances, is equal to (Bon $\left.Z_{P} / R_{P}\right)$, as known from Eq. (1). Therefore, the magnetic field due to the control coils, which is required to bring the plasma back to zero in the vertical position, is equal to - $\left(\mathrm{B}_{\mathrm{o}} \mathrm{Z} \mathrm{Z}_{\mathrm{P}} /\right.$ $\left.R_{P}\right)$, too. 


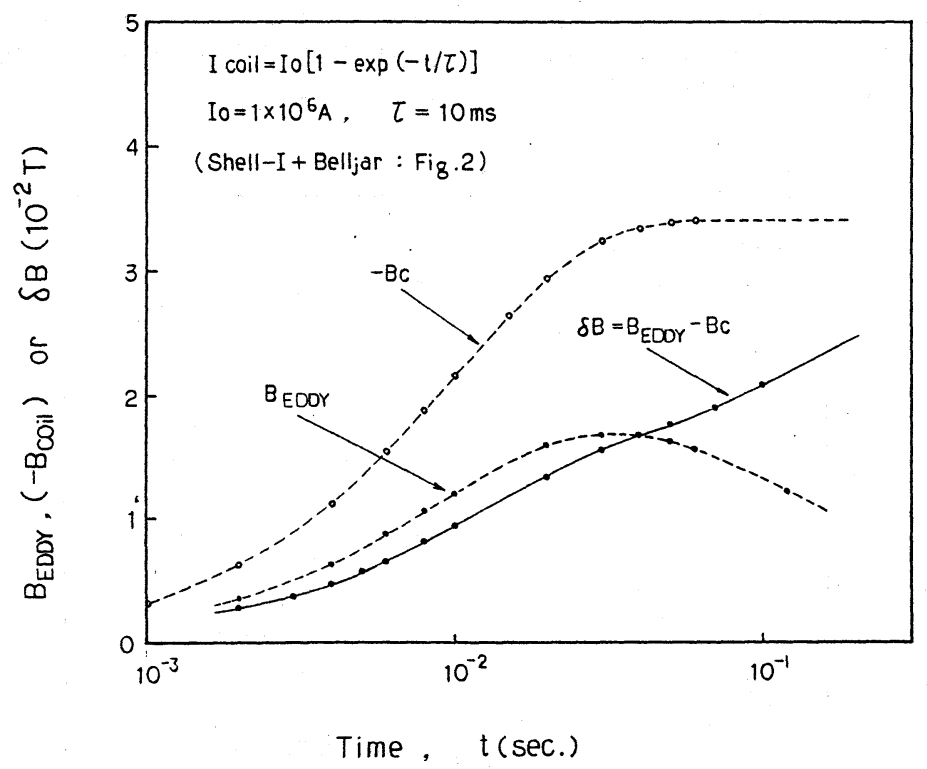

Fig. 4.7 Time evolutions of both magnetic fields due to the control coils ( $\left.\boldsymbol{B}_{\text {coil }}\right)$ and the total eddy current ( $\boldsymbol{B}_{\text {eddy }}$ ), and $\delta B\left(=|| B_{\text {coil }}|-| B_{\text {eddy }}||\right.$ at the major radius of the plasma.

In Fig. 3.7, the position of $\mathrm{Z}_{\mathrm{P}}=0$ at which the plasma counterbalances near $1 \mathrm{~ms}$ with the total magnetic field there produced, is found to have about $1.4 \mathrm{~cm}$ in the vertical coordinate and to be corresponding to $0.0018 \mathrm{~T}$ in (Bon $\left.Z_{P} / B_{P}\right)$. Then, the magnetic field strength, $2.5\left(B\right.$ on $\left.Z_{P} / R_{P}\right)$ required to give the plasma back to zero in the vertical position, attains 0.0045T. The control current in Fig. $3.7, \mathrm{Ic}$ is equal to about 13KA in the peak value and corresponds to about $0.0048 \mathrm{~T}$ in the field strength. This value is found to be nearly equal to 2.5 ( $\left.B \circ n Z_{P} / R_{P}\right)$. As known from the above-mentioned results, the analysis due to the reactor model which is described here, is found to be usefuls for understanding the results which are described in Section 3.

\section{Acknowledgement}

The authors would like to express their sincere thanks to Prof. K.Miyamoto in University of Tokyo, and Drs. T. Tone, Y. Seki, H. Iida and K. Tachikawa in Japan Atomic Energy Research Institute (JAERI), for their valuable discussion and continuing encouragement.

They also wish to thank all other members of the next reactor design group in JAERI, and Drs. A. Kameari and S. Kasai in Mitsubishi Fusion Center for useful discussion and suggestion on the design of shell structure and development and application of computer codes.

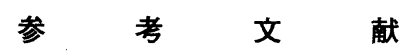

1) K. Ueda, S. Nishio, M. Sugihara, and N. Fujisawa : J. Nucl. Science and Technology, 23 [12] 1029 (1987).

2) A. Kameari, S. Niikura, and N. Fujisawa : Nucl. Eng. Des. / Fusion, 2, 365 (1985).

3) For Example, H. Yokomizo, S. Seki, A. Kitsunezaki, T. Matsuda, and R. Saito : JAER - M6693 (1976) (in Japanese). 